Claremont Colleges

Scholarship@ Claremont

All HMC Faculty Publications and Research

HMC Faculty Scholarship

11-1-1994

\title{
Rapid Relaxation of an Axisymmetric Vortex
}

Andrew J. Bernoff

Harvey Mudd College

Joseph F. Lingevitch

Northwestern University

\section{Recommended Citation}

Rapid relaxation of an axisymmetric vortex. Andrew J. Bernoff and Joseph F. Lingevitch, Phys. Fluids 6, 3717 (1994).

This Article is brought to you for free and open access by the HMC Faculty Scholarship at Scholarship @ Claremont. It has been accepted for inclusion in All HMC Faculty Publications and Research by an authorized administrator of Scholarship @ Claremont. For more information, please contact scholarship@cuc.claremont.edu. 


\title{
Rapid relaxation of an axisymmetric vortex
}

\author{
Andrew J. Bernoff and Joseph F. Lingevitch \\ Department of Engineering Sciences and Applied Mathematics, \\ Northwestern University, Evanston, Illinois 60208
}

(Received 22 January 1994; accepted 8 July 1994)

\begin{abstract}
In this paper it is argued that a two-dimensional axisymmetric large Reynolds number (Re) monopole when perturbed will return to an axisymmetric state on a time scale $\left(\mathrm{Re}^{1 / 3}\right)$ that is much faster than the viscous evolution time scale $(\mathrm{Re})$. It is shown that an arbitrary perturbation can be broken into three pieces; first, an axisymmetric piece corresponding to a slight radial redistribution of vorticity; second, a translational piece which corresponds to a small displacement of the center of the original vortex; and finally, a nonaxisymmetric perturbation which decays on the $\mathrm{Re}^{1 / 3}$ time scale due to a shear/diffusion averaging mechanism studied by Rhines and Young [J. Fluid Mech. 133, 133 (1983)] for a passive scalar and Lundgren [Phys. Fluids 25, 2193 (1982)] for vorticity. This mechanism is verified numerically for the canonical example of a Lamb monopole. This result suggests a physical explanation for the persistence of monopole structures in large Reynolds flows, such as decaying turbulence.
\end{abstract}

\section{INTRODUCTION}

Isolated nearly axisymmetric vortices are a common feature in two-dimensional (2-D) flows. They dominate the fields of two-dimensional decaying turbulence, ${ }^{1}$ and are commonly observed in the wakes of bluff bodies. ${ }^{2,3}$ Ting and his collaborators ${ }^{4,5}$ studied the advection and evolution of these vortices in an external flow in the limit of large Reynolds number; they can be thought of as viscous regularizations of point vortices.

Previously it has been argued that smooth localized concentrations of vorticity in the absence of external flow tend to approach axisymmetric states. ${ }^{6}$ Dritschel has shown for axisymmetric inviscid flows that if the concentration of vorticity is a monotonically decreasing positive function of radius, that the monopole is Lyapunoy stable. ${ }^{7}$ These results suggest that axisymmetric distributions of vorticity may be attracting asymptotic states in large Reynolds flows.

In this paper we consider the large Reynolds number $(\mathrm{Re} \gg 1)$ linear stability theory for axisymmetric monopoles whose vorticity is a positive decreasing function of radius that tends to zero exponentially. In this case the unperturbed monopole evolves on the viscous diffusion time scale $[G(\operatorname{Re})]$, and in fact will eventually evolve toward the Gaussian profile of the well-known Lamb vortex. ${ }^{4}$ The linear stability analysis is closely related to the mixing of a passive scalar by a vortex. In both instances the concentration of vorticity (passive scalar) is Lagrangian advected with the fluid velocity and diffuses. In the case of a passive scalar, a shear/diffusion mechanism studied by Rhines and Young homogenizes the concentration of passive scalar along the streamlines of the flow on a $\mathrm{Pe}^{1 / 3}$ time scale where $\mathrm{Pe}$ is the Péclet number. ${ }^{8}$ The shear/diffusion mechanism applied to vorticity perturbations is complicated by the fact that vorticity is dynamically coupled to the streamfunction. This coupling allows for a nonaxisymmetric deformation of the streamlines of the vortex. Lundgren ${ }^{9}$ has argued that, for large times, nonaxisymmetric vorticity perturbations become rapidly radially varying which causes the streamfunctionvorticity coupling to cancel at leading order. Consequently, he concludes that the shear/diffusion homogenization mechanism is qualitatively identical to the passive scalar case and that these perturbations decay on the $\mathrm{Re}^{1 / 3}$ time scale. Gilbert ${ }^{10}$ uses similar reasoning to treat the homogenization of vorticity in the far field of a strong vortex. However, these analyses are only applicable to rapidly varying vorticity perturbations.

Here we argue that almost all vorticity perturbations decay on the shear/diffusion time scale $\left[Q\left(\operatorname{Re}^{1 / 3}\right)\right]$. The exceptions to this are axisymmetric perturbations, which correspond to a small radial redistribution of vorticity, and two modes which correspond to spatial translates of the vortex. Our method for studying this problem is to first identify the axisymmetric perturbations and the two modes corresponding to spatial translates. It is shown that choosing a perturbation orthogonal to the translates is equivalent to choosing a perturbation that leaves the first moment of the vorticity fixed. We then introduce the mixing hypothesis, which states that nonaxisymmetric perturbations that leave the first moment fixed decay on a time scale $Q\left(\mathrm{Re}^{1 / 3}\right)$ due to the shear/ diffusion mechanism. A Wentzel-Kramers-Brillouin (WKB) theory is presented to verify this mechanism for a spatially rapidly varying perturbation to the monopole; this result is essentially a recasting of Lundgren's large time asymptotics. We address the issue that the WKB theory is only valid for rapid variations by numerically validating the mixing hypothesis for the canonical Lamb vortex. The numerical studies show that asymptotically as the perturbation decays it is expelled to larger and larger radii; the WKB result can be used to explain this expulsion. In the conclusions we discuss the role of this result for vortex monopoles in external flows.

\section{STABILITY THEORY}

\section{A. Axisymmetric vortices}

The 2-D Navier-Stokes equation can be written in terms of the advection and diffusion of vorticity $(\omega)$ :

$$
\omega_{t}+\mathbf{u} \cdot \nabla \omega=\operatorname{Re}^{-1} \nabla^{2} \omega,
$$


where the velocity (u) and vorticity can be related to a streamfunction in polar coordinates by

$$
\mathbf{u}=\frac{\psi_{\theta}}{r} \hat{r}-\psi_{r} \hat{\theta}, \quad \nabla^{2} \psi=-\omega .
$$

We are considering an exponentially localized distribution of vorticity which implies the velocity is decaying in the far field; this yields boundary conditions on the streamfunction, $|\mathbf{u}|=|\nabla \psi| \rightarrow 0$ as $|r| \rightarrow \infty$ which specify it up to a constant. For the WKB analysis presented below, the evolution of vorticity will be contrasted with the related problem of the advection and diffusion of the concentration of a passive scalar, $C$,

$$
C_{t}+\mathbf{u} \cdot \nabla C=\mathrm{Pe}^{-1} \nabla^{2} C,
$$

where $\mathbf{u}$ is a known incompressible velocity field and $\mathrm{Pe}$ is the Péclet number.

Any axisymmetric distribution of vorticity yields a steady inviscid solution to (1). The viscous evolution is purely diffusive as the velocity field is azimuthal leading to the vanishing of the nonlinearity; as such it evolves on the viscous diffusion time scale, $T=t /$ Re. If $\omega=W(r, T)$, $\psi=\Psi(r, T)$, their evolution is specified by

$$
W_{T}=\mathscr{L}^{0} W, \quad \mathscr{E}^{0} \Psi=-W, \quad \mathscr{L}^{0}=\partial_{r r}+(1 / r) \partial_{r} .
$$

Note that the vorticity has decoupled from the streamfunction. A solution of particular interest to this problem is the Lamb vortex ${ }^{3,4}$ which corresponds to the self-similar viscous decay of an initial point vortex,

$$
\begin{aligned}
& W(r, T)=\bar{W}(r, T) \equiv \frac{1}{4 \pi T} \exp \left(-\frac{r^{2}}{4 T}\right) \\
& \Psi(r, T)=\bar{\Psi}(r, T) \equiv-\frac{1}{2 \pi} \int_{0}^{r}\left[1-\exp \left(-\frac{r^{2}}{4 T}\right)\right] \frac{d r}{r} .
\end{aligned}
$$

Ting showed that any axisymmetric exponentially localized distribution of vorticity evolves toward a Lamb vortex with the same integrated vorticity. ${ }^{4}$

\section{B. Linear stability equations}

Consider a small disturbance to an axisymmetric vortex, such as the Lamb vortex,

$$
\begin{aligned}
& \omega=W(r, T)+\left(\sum_{n=0}^{\infty} \omega_{n}(r, t) e^{i n \theta}+\text { c.c. }\right), \\
& \psi=\Psi(r, T)+\left(\sum_{n=0}^{\infty} \psi_{n}(r, t) e^{i n \theta}+\text { c.c. }\right),
\end{aligned}
$$

where c.c. stands for the complex conjugate. The linearized equations which govern $\omega_{n}, \psi_{n}$ satisfy

$$
\begin{aligned}
& \left(\omega_{n}\right)_{t}+i n\left(\Omega \omega_{n}+\frac{W_{r}}{r} \psi_{n}\right)=\operatorname{Re}^{-1} \mathscr{Z}^{n} \omega_{n}, \\
& \mathscr{L}^{n}\left(\psi_{n}\right)=-\omega^{n}, \quad \mathscr{L}^{n}=\mathscr{L}^{0}-\left(n^{2} / r^{2}\right) .
\end{aligned}
$$

Here $\Omega=-\Psi_{r} / r$ is the angular velocity which is positive and decreasing in $r$. The boundary conditions on (9) and (10) are

$$
\psi_{n}, \omega_{n} \propto r^{n} \quad \text { as } r \rightarrow 0, \quad\left(\psi_{n}\right)_{r}, \omega_{n} \rightarrow 0 \quad \text { as } r \rightarrow \infty
$$

The first condition assures continuity of vorticity and its derivatives at the origin. The second reflects the localized nature of the disturbance; it is natural to consider exponentially localized vorticity perturbations.

Note that the evolution of a passive scalar advected by the same flow as described above can also be written in terms of Fourier modes,

$$
C=\sum_{n=0}^{\infty} C_{n}(r, t) e^{i n \theta}+\text { c.c. }
$$

which yields upon substitution into (3),

$$
\left(C_{n}\right)_{t}+i n \Omega C_{n}=\mathrm{Pe}^{-1} \mathscr{H}^{n} \omega_{n},
$$

with boundary conditions

$$
C_{n} \propto r^{n} \quad \text { as } r \rightarrow 0, \quad C_{n} \rightarrow 0 \quad \text { as } r \rightarrow \infty .
$$

This is analogous to the linearized vorticity equation with the coupling to the streamfunction suppressed.

\section{Axisymmetric perturbations}

Note first that for $n=0$ the vorticity equation (9) decouples from the streamfunction as we are looking at the axisymmetric portion of the perturbation,

$$
\left(\omega_{0}\right)_{t}=\operatorname{Re}^{-1} \mathscr{S}^{0} \omega_{0} .
$$

This corresponds to a small axisymmetric vorticity perturbation to $W(r, T)$ which will also evolve on the viscous diffusion time scale.

\section{Translation modes}

In this section we show that the evolution of $n=1$ perturbations is constrained to preserve the first moment of vorticity. The conservation of the first moment of vorticity is then related to existence of translational modes of the linearized disturbance equation. We conclude that a general $n=1$ perturbation can be decomposed into a component which corresponds to a translation of the vortex (which is nondecaying) and a component orthogonal to the translation modes (which we will argue decays on the shear/diffusion $\left(\operatorname{Re}^{1 / 3}\right)$ time scale). The translational modes are significant because they are nonaxisymmetric perturbations which do not decay through the shear/diffusion mechanism.

It is useful to note that the first moment of vorticity,

$$
\mathbf{M}=\int_{-\infty}^{\infty} \int_{-\infty}^{\infty} \omega \mathbf{r} d x d y, \quad \mathbf{r}=\langle x, y\rangle,
$$

is a conserved quantity of both the full and linearized Navier-Stokes equations, related to the conservation of momentum. ${ }^{3}$ Note that $\mathbf{M}=0$ for our base state, $W(r)$. Substituting (7) into this expression yields that

$$
M_{1}=\int_{0}^{\infty} \omega_{1} r^{2} d r
$$


must also be conserved. The first moment of the perturbation, $M_{1}$, is actually a measure of the displacement of the vortex. To see this we need to examine the structure of the linearized equation for the $n=1$ mode closely.

The translational invariance implies that displacing the base state, $W(r, T)$, by a distance $\mathbf{r}_{0}=\left\langle x_{0}, y_{0}\right\rangle$ corresponds to a linear perturbation,

$$
\mathbf{r}_{0} \cdot \nabla W=x_{0} W_{r} \cos (\theta)+y_{0} W_{r} \sin (\theta),
$$

which must be an exact solution to the linearized equations; this implies that

$$
\omega_{1}=\alpha W_{r}, \quad \psi_{1}=\alpha \Psi_{r},
$$

where

$$
\alpha=\frac{1}{2}\left(x_{0}-i y_{0}\right),
$$

is also an exact solution for the $n=1$ linearized equations (9) and (10). This exact solution provides a useful example for verifying our numerical code below.

To understand the role of this translational mode in the evolution, we rewrite the linearized equation in terms of $\omega_{1}$,

$$
\omega_{1 t}+i \mathscr{P} \omega_{1}=\operatorname{Re}^{-1} \mathscr{L}^{l} \omega_{1} \text {. }
$$

Here $\mathscr{P}$ is the inviscid linearized operator,

$$
\begin{aligned}
& \not D=\Omega+Q \\
& \ell=W_{r} \int_{r}^{\infty} \frac{d r}{r^{3}} \int_{0}^{r} d r r^{2},
\end{aligned}
$$

where the integral operator, $Q$, results from inverting $\mathscr{L}^{1}$ in (10) to obtain the streamfunction as an integral of the vorticity.

At this point we need to deal with the difficulty that $\mathscr{P}$ through $\Omega$ and $W$ is varying on the slow viscous time $T$. If we restrict ourselves to considering evolutions for $t \ll \mathrm{Re}^{-1}$, we can approximate $\mathscr{P}$ as being independent of time by considering $T$ as a parameter. This allows us to analyze the evolution as an eigenvalue problem.

If eigenmodes of the form

$$
\omega_{1}=\tilde{\omega}_{1} e^{\lambda t}
$$

are considered, Eq. (21) can be reduced to the eigenvalue problem

$$
\lambda \tilde{\omega}_{1}+i \mathscr{P}_{\tilde{\omega}_{1}}=\operatorname{Re}^{-1} \mathscr{F}^{1} \tilde{\omega}_{1},
$$

where (11) yields boundary conditions

$$
\tilde{\omega}_{1} \propto r \quad \text { as } r \rightarrow 0, \quad \tilde{\omega}_{1} \rightarrow 0 \quad \text { as } r \rightarrow \infty .
$$

Analyzing the far field leads to the conclusion that $\tilde{\omega}_{1}$ is exponentially decaying with $r$.

To analyze the properties of this eigenvalue problem we introduce an inner product,

$$
\left\langle\tilde{\omega}^{\prime}, \tilde{\omega}^{\prime \prime}\right\rangle=\int_{0}^{\infty}\left(\tilde{\omega}^{\prime}\right)^{*} \tilde{\omega}^{\prime \prime} r d r,
$$

where $*$ corresponds to complex conjugation. This allows us to introduce an adjoint problem

$$
\lambda^{*} \tilde{\omega}_{1}^{\dagger}-i \mathscr{P}^{\dagger} \tilde{\omega}_{1}^{\dagger}=\operatorname{Re}^{-1} \mathscr{L}^{\dagger} \tilde{\omega}_{1}^{\dagger},
$$

with boundary conditions,

$$
\tilde{\omega}_{1}^{\dagger} \propto r \text { as } r \rightarrow 0, \quad \tilde{\omega}_{1}^{\dagger}\left\{\begin{array}{l}
r, \quad \text { if } \lambda=0 \\
1 / r, \quad \text { if } \lambda \neq 0
\end{array} \quad \text { as } r \rightarrow \infty .\right.
$$

Here $\mathscr{P}^{\dagger}$ is the adjoint of $\mathscr{P}$,

$$
\begin{aligned}
& \mathscr{P}^{\dagger}=\Omega+\mathscr{Q}^{\dagger}, \\
& \mathscr{Q}^{\dagger}=r \int_{r}^{\infty} \frac{d r}{r^{3}} \int_{0}^{r} d r r W_{r} .
\end{aligned}
$$

The far-field boundary conditions (29) are obtained from dominant balances at large $r$; the algebraic behavior is sufficient to cause the boundary terms in the inner product to vanish when deriving the adjoint.

First consider the inviscid problem $\left(\operatorname{Re}^{-1}=0\right)$; from the stability result of Dritschel ${ }^{7}$ we know that the inviscid spectrum is pure imaginary and we expect it to be continuous. ${ }^{11}$ We can also show there is a single zero eigenvalue; this follows from solving $\mathscr{P} \tilde{\omega}_{1}^{N}=0$ to yield a unique null vector,

$$
\tilde{\omega}_{1}^{N}=W_{r} .
$$

Note that is exactly the solution generated by the translational invariance (19). Similarly, there is unique adjoint null vector which satisfies $\mathscr{P}^{\dagger}\left(\tilde{\omega}_{1}^{N}\right)^{\dagger}=0$,

$$
\left(\tilde{\omega}_{1}^{N}\right)^{\dagger}=r,
$$

which is equivalent to noting that the moment $M_{1}$ is conserved. Now, if we choose an initial condition with $M_{1}=0$, by the Fredholm alternative it will be orthogonal to the translational mode. This demonstrates the connection between the first moment and the translation mode.

For the viscous problem $\left(\operatorname{Re}^{-1} \neq 0\right.$ ), we first note that the inviscid adjoint null vector (33), remains a null vector corresponding to the conservation of $M_{1}$. The inviscid null vector is no longer a null vector for the viscous problem, although it can be used as the leading-order term in a power series expansion for the viscous null vector in powers of $\mathrm{Re}^{-1}$; the reason for this discrepancy is the suppression of the dependence on the slow time $T$. For small $\operatorname{Re}^{-1}$, setting $M_{1}=0$ can still be interpreted as enforcing orthogonality to the translational mode at leading order.

Finally, note that even if we include the slow time evolution of the vortex, a perturbation with $M_{1}=0$ initially will remain so for all time. This can be interpreted as saying that a perturbation that is initial perpendicular to the translation mode (which evolves on the slow time scale) will remain orthogonal for all time. Note that for a perturbation with $M_{1} \neq 0$, we can subtract out a multiple of the exact solution (32) to yield a problem with $M_{1}=0$; this can be interpreted as being equivalent to perturbing a slightly translated vortex.

In conclusion, what we have argued in this section is that the $n=1$ perturbation can be broken into two components; a translational mode which is nondecaying, and which vanishes with the first moment of the vorticity (a conserved quantity) and a component orthogonal to the translational mode which decays due to the shear/diffusion mechanism as will be discussed below. 


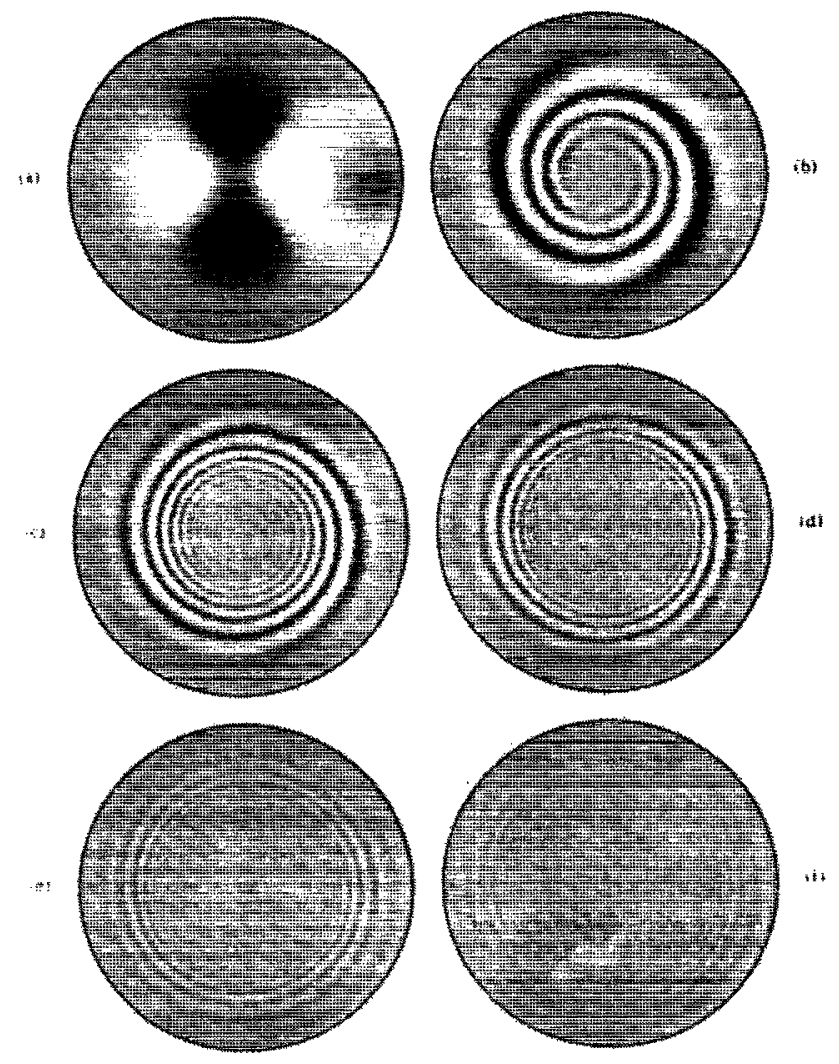

FIG. 1. Gray-scale contour plots of an $n=2$ vorticity perturbation to a Lamb vortex at $\operatorname{Re}=10^{5}$. Black (white) corresponds to a negative (positive) and gray is zero vorticity. The six frames (a) $-(\mathrm{f})$ show the vorticity a times $\tau=0$, $10,20,30,40,50$, respectively.

\section{E. The mixing hypothesis}

The basic hypothesis of this paper can now be stated: Mixing hypothesis: If the vorticity distribution $W(r, T)$ is subject to a nonaxisymmetric $\left(\omega_{0}=\psi_{0}=0\right)$ linear perturbation that preserves the first moment of vorticity $\left(M_{1}=0\right)$, this perturbation will decay on a time scale $\mathscr{O}\left(\operatorname{Re}^{1 / 3}\right)$.

The interpretation of this result is that a general perturbation to the vortex will result in a slight axisymmetric redistribution of vorticity (the $n=0$ perturbation), a slight translation of the vortex (the translational mode component of the $n=1$ perturbation), and the remainder will rapidly decay due to the mixing of vorticity through a shear/diffusion mechanism (see Refs. 8-10,12, and citations therein). A qualitative understanding of this mechanism can be had by looking at Fig. 1. Note the initial distribution of vorticity is wound into a tight spiral by differential rotation (axial shear). The rapid variation in vorticity then acts to enhance diffusion which smooths out the variations. The shear/diffusion leads to variations on a length scale $\mathrm{Re}^{-1 / 3}$ and on a time scale $\mathrm{Re}^{1 / 3}$; below we develop a WKB theory for this mechanism when vorticity is rapidly radially varying and then verify numerically that perturbations satisfying the conditions of the mixing hypothesis eventually decay in this predicted fashion.

\section{F. WKB theory}

In this section we give an argument that small-scale variations in the vorticity decay rapidly due to the shear/ diffusion mechanism. Under the assumption of rapidly varying vorticity perturbations, the streamfunction-vorticity coupling is shown to be small and the vorticity perturbation is homogenized like a passive scalar as shown by Lundgren. ${ }^{9}$ Here we recast his large time asymptotics using a WKB ansatz of a rapidly varying vorticity perturbation.

For $n \geqslant 1$, the action of advection will be to rapidly shear the vorticity into the spiral shape mode seen in Fig. 1. We can represent the solution as a complex exponential,

$$
\omega_{n}=A(r, t) e^{i \phi(r, t)}
$$

where we assume that $\phi_{r}$ is negative corresponding to $\Omega$ decreasing in $r$ and make a WKB ansatz of rapid variation, $\operatorname{Re} \gg-\phi_{r} \gg 1$. The upper limit excludes variation on the diffusive scale which we expect would decay on an order unity time scale; note this range includes the shear/diffusion scale, $\mathrm{Re}^{1 / 3}$. First we solve Eq. (10) for the streamfunction in terms of the vorticity and estimate its size in terms of the WKB ansatz (34),

$$
\psi_{n}=r^{n} \int_{r}^{\infty} r^{-(2 n+1)} d r \int_{0}^{r} r^{n+1} \omega_{n} d r \sim \frac{A}{\phi_{r}^{2}} \ll 1 .
$$

Physically this implies that the vorticity's effect on the flow field tends to cancel due to its rapid variation. This cancellation suppresses the coupling of the perturbation streamfunction to the vorticity and consequently the decay of these perturbations is identical for the passive scalar and the vorticity problems. This result generalizes Lundgren's observation ${ }^{9}$ that at large times the streamfunction decays like $t^{-2}$.

Substituting this ansatz into (9) and expanding yields

$$
\begin{aligned}
& \phi_{t}=-n \Omega+\mathscr{Q}\left(\operatorname{Re}^{-1} \phi_{r}, \phi_{r}^{-2}\right), \\
& A_{t}=-\operatorname{Re}^{-1} \phi_{r}^{2} A+\circlearrowleft\left(\operatorname{Re}^{-1} A, A \phi_{r}^{-3}\right) .
\end{aligned}
$$

Solving these equations at leading order yields,

$$
\begin{aligned}
& \phi(r, t)=\phi_{0}(r)-n \Omega t, \\
& A(r, t)=A_{0}(r) \exp \left(-\operatorname{Re}^{-1} n^{2} \Omega_{r}^{2} t^{3} / 3\right),
\end{aligned}
$$

where $\phi_{0}, A_{0}$ are determined by matching to initial data. This allows us to conclude that these rapid variations decay on a time scale $\operatorname{Re}^{1 / 3}$ in agreement with Lundgren. ${ }^{9}$

The question that remains to be answered is if all the perturbations allowed by the conditions of the mixing hypothesis eventually cascade down to these rapidly varying scales or if there is some anomalous mode with a different spatial structure and asymptotic decay rate. Note that the existence of the translation mode augurs that other such modes may exist. To answer this question we investigate the behavior numerically.

\section{NUMERICAL VERIFICATION OF THE MIXING HYPOTHESIS}

In this section we give the results of a numerical solution to the linearized disturbance equations for the Lamb vortex 
(9) and (10). We demonstrate that nonaxisymmetric disturbances that are orthogonal to the translation modes (i.e., disturbances for which $\mathbf{M}=0$ as discussed in Sec. II D) are attenuated on the $\operatorname{Re}^{1 / 3}$ time scale by the shear/viscousdiffusion mechanism discussed in Sec. II.

We solved the linearized Navier-Stokes equations (9) and (10) by a second-order splitting of the advection and diffusion operators. Second-order spatial finite differences were used; the advection step was implemented explicitly with a fourth-order Runge-Kutta scheme using a tridiagonal inversion to find the perturbation streamfunction at each step. The diffusive step was solved implicitly with a CrankNicholson scheme. To test this numerical code we used the nontrivial exact solution (19) generated by the translational invariance of the Navier-Stokes equations.

In the numerical results presented below, the slow evolution of the Lamb vortex on the Re time scale has been suppressed by taking $T=1$ in (5). This corresponds to considering perturbations to a vortex with an initial nonzero width. We note that since it is shown a posteriori that vorticity disturbances decay asymptotically faster than the spreading of a Lamb vortex we are justified in neglecting the slowly increasing width of the vortex. The mixing hypothesis has been tested with the slow spreading included and we observe that this does not qualitatively change the results presented below.

Figures $1(a)-1(f)$ are gray-scale contour plots showing the mixing of an $n=2$ vorticity perturbation to the Lamb vortex. The initial vorticity perturbation is proportional to,

$$
\omega_{2}(r, 0)=r^{2} \exp \left(-r^{2} / 4\right),
$$

and is shown in Fig. 1(a). Black (white) corresponds to a negative (positive) vorticity and gray corresponds to zero vorticity, which is shown for a circular domain of radius 6 . Figures $1(b)-1(f)$ show that on the $\operatorname{Re}^{1 / 3}$ time scale the initial vorticity distribution becomes rapidly radially varying due to the shearing along the streamlines of the unperturbed Lamb vortex. Once the initial perturbation is sheared to small enough radial scales, viscous diffusion can effectively smooth the vorticity gradients and the vorticity perturbation is homogenized. Note that as time increases the maximum amplitude of the vorticity perturbation is expelled toward larger radii due to the decrease of shear with increasing radius.

Figures 2(a) and 2(b) show the numerical data for the decay of $n=1,2$ vorticity disturbances for Reynolds numbers in the range $10^{3}-10^{6}$. We define the quantity, $I(t)$, as a measure of the root-mean-squared vorticity perturbation normalized by its initial value,

$$
I(t)=\left(\frac{\int_{0}^{\infty} r\left|\omega_{n}(r, t)\right|^{2} d r}{\int_{0}^{\infty} r\left|\omega_{n}(r, 0)\right|^{2} d r}\right)^{1 / 2} .
$$

The initial condition for the $n=1$ disturbance in Fig. 2(a) is specifically chosen to be orthogonal to the translational modes of the Lamb vortex and is proportional to

$$
\omega_{1}(r, 0)=\left(r^{2}-\frac{3 \sqrt{\pi}}{2} r\right) \exp \left(-\frac{r^{2}}{4}\right),
$$
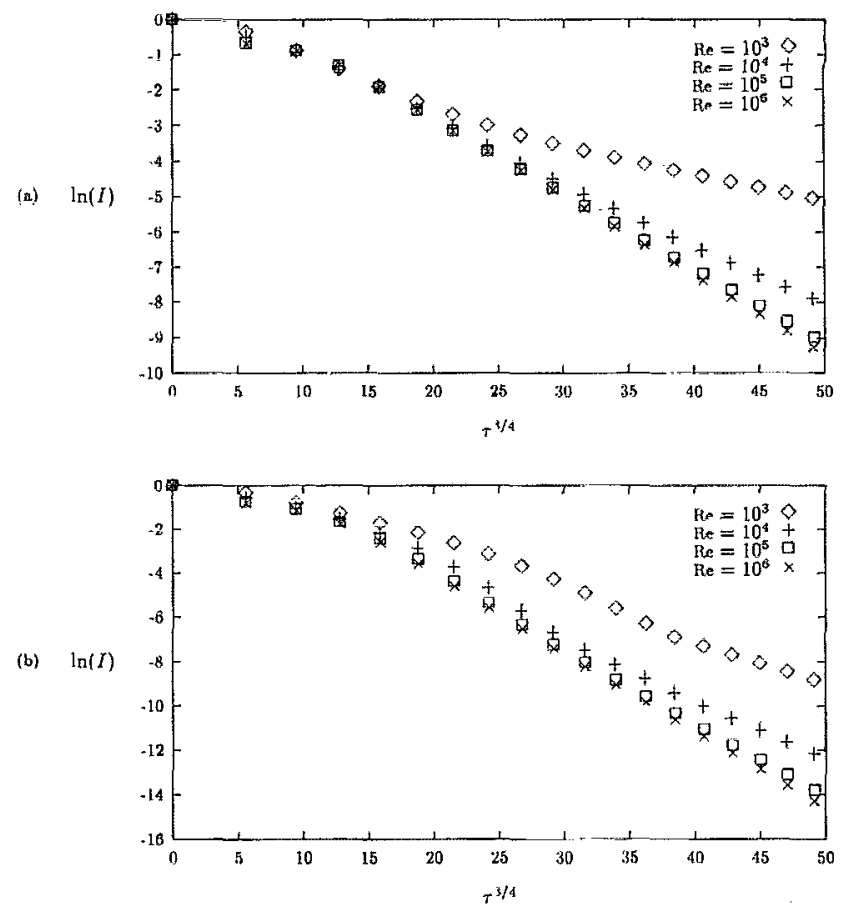

FIG. 2. Decay of root-mean-squared vorticity of a perturbation, $I$, to a Lamb vortex. For large Reynolds numbers the decay data approach an asymptotic limiting curve showing that disturbances decay on a $\mathrm{Re}^{1 / 3}$ time scale. (a) Fourier mode $n=1$ perturbation that is orthogonal to the translational modes. (b) Fourier mode $n=2$ perturbation.

and the initial condition for the $n=2$ disturbance is given by (40). Figures 2(a) and 2(b) are plots of $\ln (I)$ versus the scaled time $\tau^{3 / 4}$ and show that the perturbation decays exponentially on the $\tau=t / \mathrm{Re}^{1 / 3}$ time scale. For large times, $\ln (I)$, asymptotes to a straight line when graphed with $\tau^{3 / 4}$ as the abscissa. We explain this asymptotic behavior below as resulting from the slow decay of the vorticity perturbation in the limb of the Lamb vortex (relative to the decay in the core of the vortex). Various initial conditions satisfying the conditions of the mixing hypothesis have been tested by this numerical method and we find that all such disturbances decay in a qualitatively similar fashion to those illustrated in Figs. 2(a) and 2(b). We interpret this as numerical verification that a general nonaxisymmetric disturbance which does not translate the vortex will be sheared to small radial scales and hence decay on the $\mathrm{Re}^{1 / 3}$ time scale as shown by the WKB analysis of Sec. II F.

It is instructive to compare the decay of vorticity perturbations and the mixing of a passive scalar by a Lamb vortex. The similarity of the governing equations for these processes was discussed in Sec. II B. First we note that far from the core of the vortex, the streamline perturbation term [i.e., $\left.\left(W_{r} / r\right) \psi_{n}\right]$ in the linearized Navier-Stokes equations (9) becomes exponentially small. Neglecting this term, the disturbance equation, (9), becomes exactly the advectiondiffusion equation, (13), with vorticity replacing the concentration of the passive scalar. Thus a vorticity disturbance in the far field should be homogenized exactly analogously to a passive scalar. Figures 3(a) and 3(b) show the magnitude of a concentration (vorticity) disturbance versus 


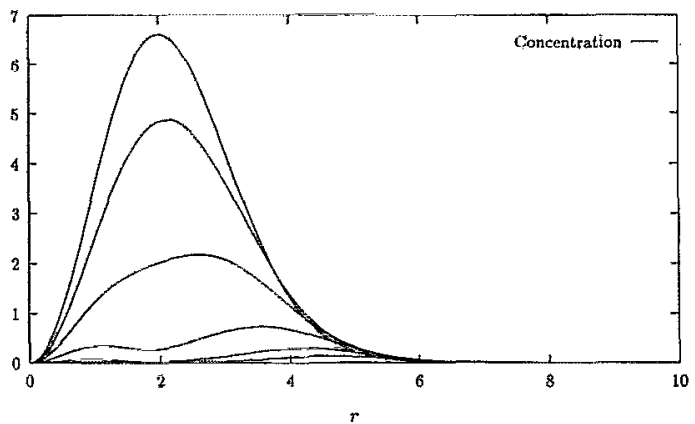

(b)

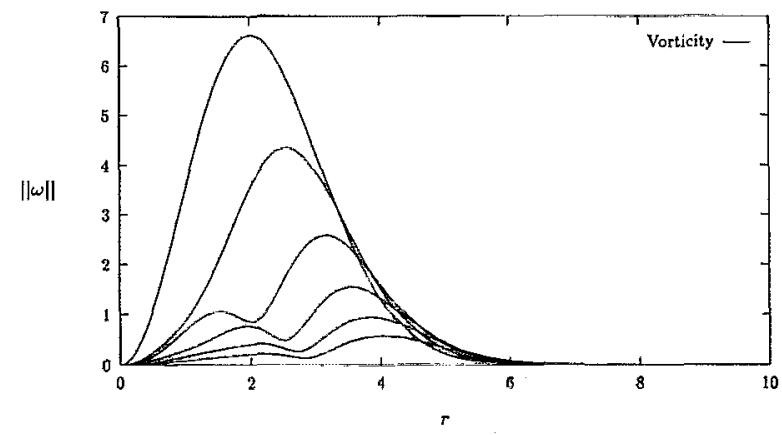

FIG. 3. Comparison of the mixing of a (a) passive scalar and (b) a vorticity perturbation by a Lamb vortex. Shown here is the modulus of the concentration (vorticity) as a function of radius for $\operatorname{Re}=10^{3}$. The initial condition is an $n=2$ Fourier mode.

radius in a Lamb vortex at five equally spaced time intervals. The initial conditions for the passive scalar and vorticity are identical. Initially the disturbance is concentrated in the core region of the vortex and the difference between the decay of vorticity and the mixing of the passive scalar is evident. The maximum of the shear rate, $\Omega_{r}$, occurs at $r \approx 1.8$. In Fig. 3(a) it can be seen that the location of most rapid homogenization coincides with the streamline for which the shear rate is maximized in agreement with the WKB analysis. Although the details of the vorticity and passive scalar mixing differ strongly within the core, the time scales for mixing are the same. For later times when the concentration (vorticity) within the core is homogenized the majority of the remaining unmixed concentration (vorticity) is in the limb of the vortex where the shear rate decays algebraically $\left(\propto 1 / r^{3}\right)$. This migration of the region of maximum unmixed vorticity toward the limb can also be observed in Fig. 1. The similarity between the mixing of a passive scalar and vorticity in the limb can be seen in Fig. 4 where the root-mean-squared concentrations (vorticity) asymptote to the same curve for large times.

As noted above and shown in Fig. 2, at large times the $\ln (I)$ data asymptotes to a straight line when graphed versus $\tau^{3 / 4}$. We have also seen (see Fig. 1) that at large times the unmixed vorticity is concentrated in the limb of the vortex where the vorticity is mixed like a passive scalar. By an argument analogous to the WKB theory described in Sec. II $F$, we have shown elsewhere ${ }^{12}$ that the nonaxisymmetric Fourier components of a passive scalar concentration will decay with the same exponential factor given in (38). Here we utilize this result and consider an initial vorticity pertur-

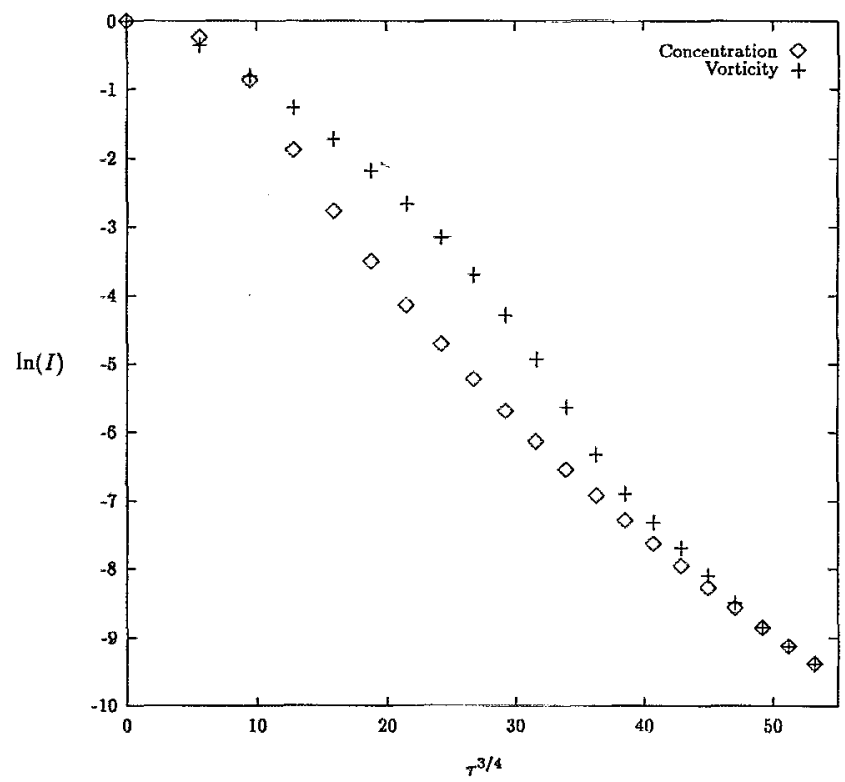

FIG. 4. Comparison of the decay of the root-mean-squared concentration of a passive scalar and vorticity for the Lamb vortex with $\operatorname{Re}=10^{3}$ and the same initial condition as Fig. 3. At large times the data asymptotes to the same curve because concentration (vorticity) in the limb of the vortex is mixed like a passive scalar.

bation, $\omega_{n} \sim r^{n} \exp \left(-r^{2} / 4\right)$. Substituting $\Omega_{r}=-1 /\left(\pi r^{3}\right)$, the far-field shear rate, into (38) gives an expression for the decay of the far-field vorticity,

$$
\begin{aligned}
& \omega_{n} \sim r^{n} \exp [-f(r, t)], \\
& f(r, t)=\left(\frac{r^{2}}{4}+\frac{n^{2} t^{3}}{3 \pi^{2} r^{6} \operatorname{Re}}\right) .
\end{aligned}
$$

Using (43) in (41), the leading-order contribution to the integral $I(t)$ at large times is obtained by applying Laplace's method. The maximum in the vorticity distribution occurs at

$$
r=r^{*}=(2 n / \pi)^{1 / 4} \tau^{3 / 8},
$$

which we note is monotonically increasing in time. At leading order the root-mean-squared vorticity is approximated by

$$
\ln (I) \sim-\sqrt{\frac{2 n}{\pi}} \tau^{3 / 4}+\Theta(n \ln \tau) .
$$

Figure 5 shows the numerical data for the decay of vorticity perturbations with Fourier modes $n=2,3,4$ and Reynolds number $\operatorname{Re}=10^{6}$. The slopes of the long time asymptotes to the numerically computed decay data in Fig. 5 are -0.345 , $-0.417,-0.474$ for $n=2,3,4$ which compare with the corresponding values of $-0.375,-0.461,-0.532$ given by (46). The differences between the asymptotic and numerical values scale with $n$ in agreement with the logarithmic corrections to (46).

\section{CONCLUSIONS}

The observation of axisymmetric vortex monopoles in large Reynolds number fluid flows leads to the speculation about the stability of such solutions to the Navier-Stokes 


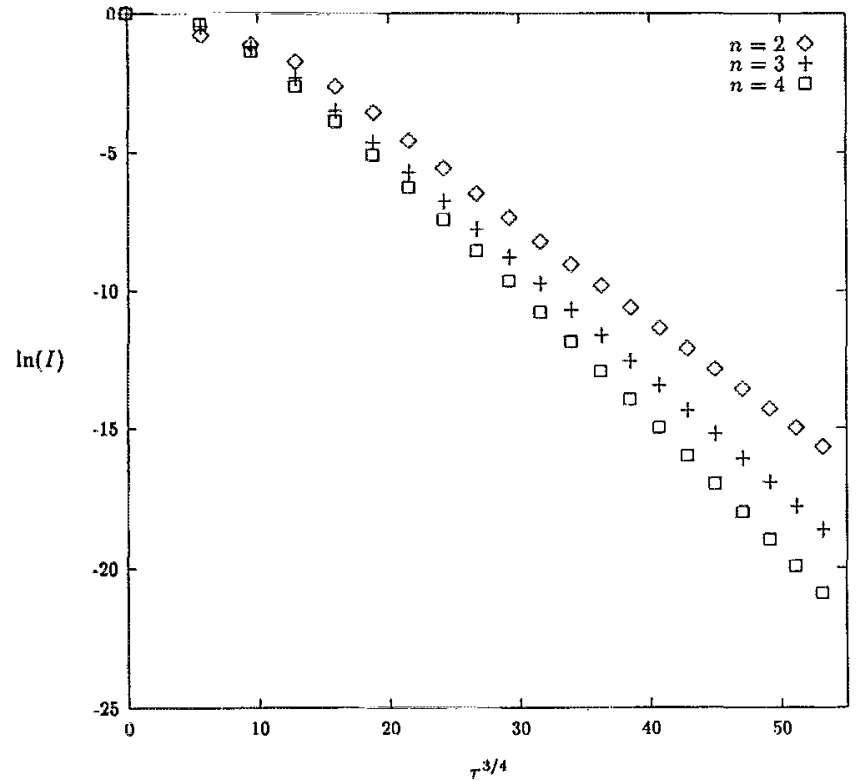

FIG. 5. Comparison of the decay of the root-mean-squared vorticity for three different Fourier modes $(n=2,3,4)$. The Reynolds number is $\operatorname{Re}=10^{6}$ and the initial conditions are $\omega_{n} \propto r^{n} \exp \left(-r^{2} / 4\right)$. At large times the data asymptotes to straight lines with logarithmic corrections.

equations. In this paper we have examined the stability of an isolated Lamb vortex to linear disturbances. By a WKB argument we reproduce the result of Lundgren that nonaxisymmetric rapidly varying vorticity perturbations to a circular vortex monopole decay rapidly $\left[Q\left(\mathrm{Re}^{1 / 3}\right)\right.$ time scale] compared to the viscous evolution time scale $[\Theta(R e)]$. A numerical solution of the linearized equations for a Lamb yortex demonstrates that almost all nonaxisymmetric initial conditions become rapidly varying due to velocity shear in the vortex whence they are effectively dissipated by viscous diffusion on the $\mathrm{Re}^{1 / 3}$ time scale. This is the same mechanism that enhances the mixing of a passive scalar within a vortex. The exceptions to this mixing hypothesis are the vorticity perturbations which correspond to infinitesimal translations of the vortex monopole. Perturbations of this type are not attenuated; they evolve on the viscous time scale and are related to conservation of the first moment of vorticity in the Navier-Stokes equations.

This work has implications for the understanding of localized vorticity distributions in external flows. In particular, it forms the foundation of our study of the viscous regularization of a point vortex. ${ }^{13}$ Previously, Ting and his collaborators $^{4,5}$ have investigated the regularization of a point vortex in a slow spatially varying irrotational flow by including the effects of a small viscosity; however, they failed to consider the shear/diffusion mechanism discussed here. We are presently using the results of this paper to extend their theory for the evolution of a monopole in a background irrotational flow (e.g., straining flow) at large Reynolds numbers. The irrotational flow causes the vortex to propagate with a velocity determined by the conservation of the first moment of vorticity. Nonuniformities in the irrotational flow allow for the distortion of the vortex from an axisymmetric state. In addition, we are also numerically studying the nonlinear stability of the monopole (with Dr. L. Rossi). We also hope to extend these ideas to study the stability of large Reynolds number axisymmetric vortex filaments.

\section{ACKNOWLEDGMENTS}

We would like to acknowledge the insightful and thorough comments of the referees to this paper, particularly in directing us to the work of Lundgren and Gilbert. This research has been supported by the National Science Foundation, Grant No. CTS-9206828. One of us (AJB) would like to thank the 20th St. Shelter for Wayward Mathematicians for its spiritual support. The other (JFL) would like to acknowledge the inspiring artwork of REL.

${ }^{1} \mathrm{~J}$. C. McWilliams, "The emergence of isolated coherent vortices in turbulent flow," J. Fluid Mech. 146, 21 (1984).

${ }^{2}$ Y. Couder and C. Basdevant, "Experimental and numerical study of vortex couples in two-dimensional flows," J. Fluid Mech. 173, 225 (1986).

${ }^{3}$ P. G. Saffman, Vortex Dynamics (Cambridge University Press, Cambridge, 1992).

${ }^{4}$ L. Ting and R. Klein, Viscous Vortical Flows (Springer-Verlag, Berlin, 1991).

${ }^{5} \mathrm{~L}$. Ting and C. Tung, "Motion and decay of a vortex in a nonuniform stream," Phys. Fluids 8, 1039 (1965).

${ }^{6} \mathrm{M}$. V. Melander, J. C. McWilliams, and N. J. Zabusky, "Axisymmetrization and vorticity-gradient intensification of an isolated two-dimensional vortex through filamentation," J. Fluid Mech. 178, 137 (1987).

${ }^{7}$ D. G. Dritschel, "Nonlinear stability bounds for inviscid two-dimensional, parallel or circular flows with monotonic vorticity, and the analogous quasi-geostrophic flows," J. Fluid Mech. 191, 575 (1988).

${ }^{8} \mathrm{P}$. B. Rhines and W. R. Young, "How rapidly is a passive scalar mixed within closed streamlines?," J. Fluid Mech. 133, 133 (1983).

${ }^{9}$ T. S. Lundgren, "Strained spiral vortex model for turbulent fine structure," Phys. Fluids 25, 2193 (1982).

${ }^{10}$ A. D. Gilbert, "Spiral structures and spectra in two-dimensional turbulence," J. Fluid Mech. 193, 475 (1988).

${ }^{11}$ P. G. Drazin and W. H. Reid, Hydrodynamic Stability (Cambridge University Press, Cambridge, 1981).

${ }^{12} \mathrm{~J}$. F. Lingevitch and A. J. Bernoff, "Advection of a passive scalar by a vortex couple in the small diffusion limit," J. Fluid Mech. 270, 219 (1994).

${ }^{13}$ J. F. Lingevitch and A. J. Bernoff, "Distortion and evolution of a localized vortex in an irrotational flow" submitted to Phys. Fluids. 